\title{
Frontières
}

\section{Construction identitaire et résilience en réadaptation}

\section{Patrick Fougeyrollas et Claire Dumont}

Volume 22, numéro 1-2, automne-printemps 2009-2010

\section{Résilience et deuil}

URI : https://id.erudit.org/iderudit/045023ar

DOI : https://doi.org/10.7202/045023ar

Aller au sommaire du numéro

Éditeur(s)

Université du Québec à Montréal

ISSN

1180-3479 (imprimé)

1916-0976 (numérique)

Découvrir la revue

Citer cet article

Fougeyrollas, P. \& Dumont, C. (2009). Construction identitaire et résilience en réadaptation. Frontières, 22(1-2), 22-26. https://doi.org/10.7202/045023ar

\section{Résumé de l'article}

Cet article présente le concept de résilience comme réaction à une rupture vécue au cours de l'existence ainsi qu'en relation avec le processus de construction identitaire. Cette réaction est conditionnée par l'interaction entre les caractéristiques intrinsèques de la personne, ses occupations ainsi que des facteurs extrinsèques ou environnementaux. La résilience est présente quand la personne vit un processus de construction identitaire favorable en dépit des obstacles et des ruptures. Elle peut même être renforcée par eux. En réadaptation, la construction identitaire peut constituer une cible d'intervention ancrée dans les composantes porteuses de sens pour la personne. En fonction du modèle du Processus de production du handicap, il est possible d'agir sur une ou plusieurs composantes, soit la personne, l'environnement et la participation sociale ainsi que sur leurs interactions, pour favoriser la résilience et la construction identitaire.
Ce document est protégé par la loi sur le droit d'auteur. L'utilisation des services d'Érudit (y compris la reproduction) est assujettie à sa politique d'utilisation que vous pouvez consulter en ligne.

https://apropos.erudit.org/fr/usagers/politique-dutilisation/ 


\section{Résumé}

Cet article présente le concept de résilience comme réaction à une rupture vécue au cours de l'existence ainsi qu'en relation avec le processus de construction identitaire. Cette réaction est conditionnée par l'interaction entre les caractéristiques intrinsèques de la personne, ses occupations ainsi que des facteurs extrinsèques ou environnementaux. La résilience est présente quand la personne vit un processus de construction identitaire favorable en dépit des obstacles et des ruptures. Elle peut même être renforcée par eux. En réadaptation, la construction identitaire peut constituer une cible d'intervention ancrée dans les composantes porteuses de sens pour la personne. En fonction du modèle du Processus de production du handicap, il est possible d'agir sur une ou plusieurs composantes, soit la personne, l'environnement et la participation sociale ainsi que sur leurs interactions, pour favoriser la résilience et la construction identitaire.

Mots clés: résilience - identité participation - occupations - réadaptation.

\section{Abstract}

This article presents the concept of resilience as a response to a rupture experienced during the existence of a person, in relation with the process of identity construction. This response is conditioned by the interaction between the person's intrinsic characteristics, his/her social participation, and the environmental factors. Resilience is present when the person experiences a process of identity construction or reconstruction in spite of obstacles and ruptures. He/she can even be strengthened by them. In the rehabilitation process, the identity construction may consist of a target of intervention anchored in the features that are meaningful to the person. According to the Disability Creation Process model, it is possible to intervene on one or several features related to the person, the environment, the social participation, as well as on their interactions, in order to promote resilience and identity construction.

Keywords: resilience - identity -

involvement - occupations - rehabilitation.

\section{Construction identitaire et résilience en réadaptation}

\section{Patrick Fougeyrollas, Ph. D., directeur, Direction de l'enseignement et du soutien scientifique, Institut de réadaptation en déficience physique de Québec. \\ Claire Dumont, erg., Ph.D., agente de recherche, Direction de l'enseignement et du soutien scientifique, Institut de réadaptation en déficience physique de Québec.}

Comment pouvait-on vivre avant la mode de la résilience? Comment pouvait-on dire: «ça va mieux»? Le concept de résilience est vaste. Il est proposé de différentes manières, parfois vagues et assez mystérieuses, pour faire jaser de nombreuses sentinelles du développement des connaissances scientifiques et populaires. Tant jaser, que ces multiples définitions quelque peu contradictoires pourraient nous perdre, et surtout nous faire perdre le fil de ce que c'est.

Un concept aussi riche de potentiel ne peut être situé, discuté et compris qu'à travers un modèle explicatif organisationnel, un cadre conceptuel susceptible d'ordonner ses multiples facettes et la dynamique temporelle de son processus de production.

Cet article tente donc de mettre en scène la construction des composantes de l'identité de chaque être humain, et ce, tout au long de sa vie. Et de montrer comment des ruptures traumatiques (maladie, accident, perte d'emploi, échec, violence, guerre, cataclysme, déménagement, mort d'un être cher, etc.) (Denham, 2008; Rousseau et al., 2003; Gibson, 2002), chocs du présent dans l'instant, ouvrent des parenthèses dans ce qui habituellement nous apparaît comme un flux quasi stable où l'on surfe sur notre quotidien, où nos actions sont en cohérence, où tout est habituel, quoi.

Quelque chose qui vous fait perdre conscience de vous-même, un événement inattendu, une rupture, un drame, un éclatement de vos assises, de vos ancrages, de vos repères. Impossible de respirer, tout simplement sidéré, à l'image de 
LA RÉSILIENCE, C'EST CE QUI SE MET EN BRANLE QUAND ON N'EST

PAS MORT, QUE CE SOIT PHYSIOLOGIQUEMENT, PSYCHOLOGIQUEMENT

OU SYMBOLIQUEMENT. QUELQUE CHOSE QUI FAIT QUE L'ON VIT

ET QUE L'ON DÉSIRE VIVRE.

la petite funambule en perte d'équilibre sur son fil dans la tempête. Va-t-elle tomber? Va-t-elle mourir? Mais le bout du chausson pousse sur la corde, l'épouse, cumulateur d'énergie, un autre pas, encore un autre, seul maintenant existe, le pilote automatique. C'est arrivé comme une grande gifle, mais elle n'est pas tombée, de l'air est passé, corps rouleau compressé, mental en compote, plus de son, aveugle. Elle avance dans le vide.

Et alors, dis-moi, petite funambule, ce que tu as pensé, quand tu as vu au sortir du brouillard, cette main qui t'a hissée sur la terre ferme: ce qui est important, répondit-elle, c'est qu'on n'est pas mort...

La résilience, c'est ce qui se met en branle quand on n'est pas mort, que ce soit physiologiquement, psychologiquement ou symboliquement. Quelque chose qui fait que l'on vit et que l'on désire vivre. Peutêtre simplement parce qu'il n'y a rien de pire que la mort et que la vie, sous toutes ses formes, a toujours constitué un combat. Mais qui est ce «on »? C'est ce que chacun d'entre nous appelle «je». La résilience repose dans ce «qui suis-je»?

\section{LA RÉSILIENCE ET L'IDENTITÉ: CONCEPTS ET ENJEUX POUR DIFFÉRENTES POPULATIONS}

La construction d'une identité et la résilience font partie du développement humain, que plusieurs auteurs situent principalement à l'adolescence, mais il est généralement admis que le développement humain se poursuit pendant toute la vie et que la résilience constitue une ressource pour faire face à différentes situations problématiques (Dumont et al., 2006; Gibbons et al., 2006). Les caractéristiques intrinsèques de l'individu, l'environnement (physique, social, culturel, incluant certaines personnes significatives ainsi que les événements) et l'occupation (pris dans un sens large, comme étant l'ensemble des activités et habitudes de vie) sont les éléments majeurs de la construction de l'identité (Dumont et al., 2006; Deeny et al., 2005). La résilience, quant à elle, est le résultat de l'interaction entre ces éléments de manière à les renforcer mutuellement et à développer une identité cohérente et forte, pouvant faire face à l'adversité (Cummings et al., 2005 ; Dumont, 2007). Une personne peut démontrer de la résilience par sa manière de réagir aux événements contraignants, en étant même renforcée par eux. Un environnement peut être qualifié de résilient s'il demeure stable malgré les assauts qu'il subit et une occupation peut être considérée comme résiliente si elle peut être ajustée à différentes situations et personnes tout en conservant sa finalité.

Afin d'illustrer des applications du concept de résilience, plusieurs études récentes qui ont porté sur la résilience et l'identité ont été répertoriées. Ces études se sont intéressées à diverses populations que l'on pourrait qualifier de «différentes», «minoritaires», ou «vivant des contraintes» en fonction de différentes caractéristiques (race, origine ethnique, religion, orientation sexuelle, incapacités, âge, niveau socioéconomique, milieu de vie et autres). Souvent dans ces diverses études, les personnes ne pouvaient s'identifier à la majorité en place, qu'elle fût de race blanche, de religion chrétienne, de milieu socioéconomiquement aisé ou autres. Pour les auteurs de ces études, la résilience et le développement de l'identité font partie d'un même processus (Barrow et al., 2007 ; Ferrer et al., 2007 ; TummalaNarra, 2007; Ungar et al., 2007).

Parmi les facteurs d'identification et de résilience analysés, la religion constitue un élément important dans plusieurs populations (Yick, 2008) de même que l'origine ethnique. Ainsi, les personnes de religion musulmane peuvent vivre des difficultés d'identification, notamment en raison des conflits armés au MoyenOrient et du terrorisme causé par certains sous-groupes (Nguyen-Gillham et al., 2008; Coleman et al., 2007). Différentes populations d'origines ethniques variées et vivant aux États-Unis ont été confrontées à des difficultés d'identification, notamment à cause de la discrimination: les personnes de race noire (Hall et al., 2007; Shin et al., 2007), les hispanophones (Ong et al., 2006; Holleran et al., 2003), les immigrants (Lee, 2005) et autres. Des situations équivalentes sont également présentes au Canada (Sundar, 2008) et dans d'autres pays (Nikora et al., 2007). Face à la discrimination, le renforcement identitaire des minorités contribue à leur bien-être et à leur prospérité. La réussite des immigrants qui subissent de la discrimination est généralement associée à la résilience. L'autonomisation (empowerment) est une autre stratégie proposée qui va dans le même sens (Dumont, 2007; Querimit et al., 2003).

L'orientation sexuelle est également un élément qui a été considéré dans la construction identitaire et qui peut conduire à une marginalisation. Ainsi, les jeunes dont l'orientation sexuelle n'est pas définie vivent des difficultés d'identification qui peuvent causer plusieurs problèmes de comportement (Rose et al., 2006; Harrison, 2003). Les gais et les lesbiennes sont susceptibles de vivre des problèmes de santé mentale qui peuvent être associés à un problème d'identification et à un manque de résilience (Riggle et al., 2008; Scourfield et al., 2008).

Plusieurs autres types de "différences» ont été étudiés sous l'angle de l'identité et de la résilience: les pauvres (Burney et al., 2008), les aînés (Schlossberg, 2009), les jeunes décrocheurs ou marginaux (Bottrell, 2007; Brooke, 2006), les familles dont un membre a une déficience intellectuelle (Grant et al., 2007), les enfants victimes de violence (Graham-Bermann et al., 2004), les personnes porteuses de VIH-sida (Bletzer, 2007) et autres. Dans plusieurs situations, les auteurs associent la résilience à un processus d'adaptation et de reconstruction identitaire tel que vécu dans d'autres contextes (Dumont et al., 2006; Deeny et al., 2005).

Pour toutes ces populations, la résilience et la construction ou la reconstruction identitaire étaient des éléments pouvant améliorer leurs conditions de vie, leur qualité de vie, leur bien-être physique ou psychologique. Elles rencontraient des obstacles, vivaient de la discrimination, des oppressions ou des contraintes qui les ont conduites à développer une nouvelle identité qui leur est propre, sans avoir désormais besoin de s'identifier à une majorité, dans une perspective de croissance personnelle et d'affirmation de soi. Être confronté et surmonter des obstacles pour en sortir plus fort constitue la résilience. En de nombreux points, ces personnes vivaient un processus qui s'apparente à celui vécu par les personnes ayant des déficiences physiques persistantes et significatives et qui vivent des situations de handicap. En effet, les personnes qui ont des incapacités vivent un processus de construction ou de reconstruction identitaire en passant à travers un processus d'adaptation qui a été décrit par de nombreux auteurs et a fait l'objet de plusieurs études (Dumont et al., 2007 ; Dumont et al., 2006; Fougeyrollas, 2004). Les prochains paragraphes approfondissent cette situation particulière. 


\section{L'APPORT DU MODĖLE DU PROCESSUS DE PRODUCTION DU HANDICAP À LA COMPRÉHENSION DE LA RÉSILIENCE}

Le cadre conceptuel du Processus de production du handicap ( $\mathrm{PPH})$ (Fougeyrollas et al., 1998a; Fougeyrollas et al., 1998b ; Fougeyrollas, 1996) est fondé sur un modèle systémique de développement humain. Celui-ci met l'accent sur la relation interactive entre les facteurs personnels, incluant les facteurs identitaires, les facteurs environnementaux et ce que les personnes font de la naissance jusqu'à la fin de leur vie.

Le développement humain est ainsi défini comme une interaction, une coadaptation réciproque, dynamique, dans le temps, tout d'abord du corps avec ses systèmes organiques et ses aptitudes fonctionnelles influencées par une matrice relationnelle culturelle, c'est-à-dire un ensemble de variables environnementales qui donnent un sens, une identité sociale, par la médiation de facteurs de stimulation, de protection, de facilitateurs et de facteurs de risque, d'inhibition, de contraintes ou d'obstacles à la réalisation d'activités sociales. Ce que nous nommons les habitudes de vie composées d'activités courantes et de rôles sociaux.

La contribution du PPH est de définir la participation sociale comme un résultat situationnel de l'interaction personne/ environnement et non comme une capacité de la personne. La qualité de participation sociale ne peut être déduite des caractéristiques corporelles et fonctionnelles spécifiques de chacun et surtout pas uniquement de son profil de déficiences et d'incapacités. Elle doit impérativement prendre en compte les facteurs identitaires. Elle correspond à un ensemble de possi-

\section{LA RÉSILIENCE EST LE RÉSULTAT}

\section{DE LA DYNAMIQUE INTERACTIVE}

\section{D'UNE CONSTRUCTION}

IDENTITAIRE COHÉRENTE.

bilités plus ou moins étendues de réalisation d'activités composant ou construisant l'expérience de vie sociale singulière de chacun d'entre nous.

Le modèle de développement humain permet donc de comprendre et d'expliquer le processus de production, tout autant que de prévention du handicap, comme un flux dynamique de l'instant présent. Une avancée continue de construction issue de la coïncidence, de l'interaction entre cet être particulier et ce qu'il fait ou ce qui lui arrive dans un contexte particulier.

L'identité, d'un point de vue synchronique, c'est comme les multiples pelures d'un oignon qui croît de l'intérieur, à partir de l'empreinte génétique branchée sur tous ses ancêtres, par définition, des gagnants. L'identité est sculptée de tous les gestes, affects, nourritures qui ont permis à notre conscience d'émerger, jusqu'au langage, jusqu'à ce que le miroir nous donne un nom et la possibilité de survivre, de nous construire jusqu'à ce que «je» puisse se dire et se reconnaître (Fougeyrollas, 2004). C'est dans ce très long façonnement que certains éléments, certains ancrages, certaines images, certaines sensations, font que nous avons le désir de vivre. C'est ce qui donne sens à notre prochain pas sur le fil de l'existence. Chacun d'entre nous a vécu cette construction identitaire mais comme notre empreinte génétique et notre modelage culturel, chacune est unique et indécryptable par autrui. Voilà ce dont tente de parler la résilience.

\section{CONTRIBUTION DE LA PRISE EN COMPTE DE LA CONSTRUCTION IDENTITAIRE DANS LE PROCESSUS DE RÉADAPTATION}

La résilience est le résultat de la dynamique interactive d'une construction identitaire cohérente. Elle permet de comprendre que le développement du potentiel biologique et des capacités fonctionnelles ne suffit pas lorsque l'on désire définir des objectifs ou stratégies de réadaptation sans y introduire la construction identitaire de chaque personne. Autant nous avons mis l'accent depuis les années 1980 et 1990, sur les plans théorique et clinique, sur la perspective écologique, sur la nécessité de prise en compte de la qualité des facteurs environnementaux dans la définition du projet de vie, des habitudes de vie valorisées et ayant un sens pour la personne, autant il est important de tenir compte de ce qui constitue son identité propre (Fougeyrollas et al., 2007; Fougeyrollas et al., 2002; Fougeyrollas et al., 2001; Fougeyrollas et al., 1998a; Fougeyrollas, 1996). Le phénomène du développement de l'intérêt pour la prise en compte de la résilience est donc étroitement lié à la compréhension de la nécessité de mieux cerner les composantes de l'identité et de ce qui fait sens pour chacun dans l'aventure de reconstruction d'un nouveau «je » à partir des ancrages prémorbides qui font que le survivant vit.

Les facteurs identitaires sont, bien sûr, le genre, l'âge, l'appartenance à un groupe ethnique, la scolarité et les caractéristiques socioéconomiques. Mais ils font aussi référence au sens, à la signification que nous donnons à notre expérience de vie, à notre imaginaire, rêves et croyances, à nos réussites et difficultés dans ce que nous avons la possibilité de faire. Ce "possible» est donc étroitement lié aux possibilités de participation sociale et de succès de développement de notre potentiel humain au sein de notre contexte culturel.

Donc, avec la réadaptation, il faut faire quelques distinctions pour une utilisation du concept de résilience, en considérant notamment l'impact des facteurs environnementaux qui conditionnent le possible.

Comme tout être humain, une personne naissant avec des différences de potentiel de développement organique ou fonctionnel, ou les acquérant en bas âge, ne vit pas de rupture mais construit son identité et développe sa résilience en fonction de la spécificité de l'interaction dans le temps des trois grandes dimensions: la dimension personnelle, son contexte de vie et la qualité de participation sociale dont elle va pouvoir faire l'expérience.

L'intervention d'adaptation doit ici, dans une approche individualisée, tenir compte de ces trois dimensions construisant la résilience ainsi que des interactions entre ces dimensions. On peut ici intervenir autant sur le développement du potentiel fonctionnel que sur l'environnement et les situations de réalisation d'habitudes de vie qui renforceront les ancrages et succès identitaires de la personne.

Dans le cas d'une rupture traumatique entraînant une atteinte organique ou symbolique et des incapacités physiques ou mentales, les caractéristiques identitaires acquises, dont la qualité de résilience de la personne, comme ses valeurs, croyances, ses autoreprésentations historiques de succès ou d'échecs deviennent des éléments à prendre soigneusement en compte au même titre que les dimensions organiques, psychologiques et fonctionnelles, la qualité de l'environnement physique et social et les habitudes de vie valorisées par la personne et son milieu. C'est la prise en compte de tous ces éléments du système qui permet de créer ou de proposer des situations de participation sociale favorisant la résilience par la reconstruction identitaire, du sens donné au projet de vie, par l'estime de soi, l'expérience du succès, les petits progrès. On peut proposer des soutiens et facilitateurs ou l'apprentissage de stratégies gagnantes pour contourner ou surmonter les obstacles environnementaux et les incapacités fonctionnelles mises en jeu dans chaque habitude de vie spécifique. 
Traditionnellement, l'intervention en réadaptation vise à réduire les déficiences et les incapacités et à favoriser l'autonomie dans les habitudes de vie et la participation sociale. Bien sûr, ces interventions ont toujours leur place. Toutefois, selon la réflexion proposée ici, l'intervention pourrait consister à rechercher ou créer des environnements favorables, ceux qui auront le plus de potentiel pour faire vivre des succès à la personne et lui permettre de se développer. Un environnement favorable n'est pas nécessairement exempt d'obstacles. Certains obstacles peuvent en effet contribuer au développement de la résilience de la personne. Une partie de l'art de la réadaptation pourrait ainsi consister en la réussite du dosage subtil de soutien et de défi approprié à la personne et à la situation pour qu'elle poursuive son développement. Par exemple, l'intégration d'une personne ayant des incapacités dans un milieu scolaire ou un milieu de travail peut sembler faire surgir certains obstacles à première vue. Nombreuses sont les situations toutefois qui ont démontré que cette intégration a été bénéfique non seulement pour la personne ayant des incapacités, mais pour les personnes qui fréquentaient ce milieu ainsi que le milieu lui-même. On peut mentionner entre autres la productivité globale augmentée, l'amélioration de la coopération et de l'entraide qui contribue à un meilleur succès, un climat de travail plus serein, des attitudes plus respectueuses et plus ouvertes. Du point de vue des interactions, il serait ici question de synergie: tous bénéficient de la présence de l'autre.

La résilience est le résultat d'une interaction dans un contexte écosystémique (Dumont, 2007; Waller, 2001). Contrairement à la chaîne de causalité qui tend à prédire le résultat d'une action, le résultat d'une interaction reste imprévisible. Quand on agit sur l'individu, l'environnement ou les habitudes de vie, le résultat de l'interaction va dépendre de la manière dont les autres composantes vont réagir. Diverses combinaisons sont possibles à partir de ces trois composantes. Par exemple, avec les mêmes caractéristiques personnelles, une identité cohérente peut se construire dans un environnement donné, mais pas dans un autre, avec une occupation donnée et pas une autre et ainsi de suite. L'intervention peut ainsi consister à rechercher les combinaisons gagnantes (Dumont, 2007).

En résumé, il est nécessaire de distinguer, d'une part, le développement de la résilience comme résultat identitaire de l'interaction entre les facteurs personnels, environnementaux et la participation sociale qui est commune aux personnes avec ou sans incapacités et liée au processus de développement humain, d'adaptation humaine indissociable de sa matrice culturelle. Et, d'autre part, la prise en compte de la résilience comme variable du processus de reconstruction identitaire et de réadaptation dans la définition d'un projet de vie qui a du sens. Il faut alors cerner ce qui constitue un moteur pour aller de l'avant dans la vie, pour que l'oignon puisse continuer à croître en se nourrissant de certaines certitudes, de certaines couches de sens associées au succès, à l'efficacité personnelle, à la gratification, à la reconnaissance. Cela permet alors la définition de modes d'intervention sur les capacités et les facteurs environnementaux dynamisés par ses ancrages de reconstruction d'une nouvelle identité et mis en jeu dans la réalisation d'objectifs de vie valorisés ou constituant un enjeu signifiant pour la personne.

\section{Bibliographie}

BLETZER, K.V. (2007). «Identity and resilience among person with HIV: A rural African American experience », Qualitative Health Research, vol. 17, no ${ }^{\circ}$ 2, p. 162-175.

BOTTRELL, D. (2007). «Resistance, resilience and social identities: Reframing "problem youth" and the problem of schooling », Journal of Youth Studies, vol. 10, $\mathrm{n}^{\circ} 5$, p. 597-616.

BROOKE, S.L. (2006). « Building resiliency with at-risk youth», PsycCritiques, vol. 51, $\mathrm{n}^{\circ} 42$.

BURNEY, V.H. et J.R. BEILKE (2008). "The constraints of poverty on high achievement », Journal of the Education of the Gifted, vol. 31, n 3, p. 295-321.

COLEMAN, P.T. et J.K. LOWE (2007). "Conflict, identity, and resilience: Negotiating collective identities within the Israeli and Palestinian diasporas ", Conflict Resolution Quarterly, vol. 24, nº 4, p. 377-412.

CUMMINGS, G.S. et al. (2005). "An exploratory framework for the empirical measurement of resilience », Ecosystems, vol. 8, $n^{\circ} 8$, p. 975-987.

DEENY, P. et B. MCFETRIDGE (2005). "The impact of disaster on culture, self, and identity: Increased awareness by health care professionals is needed", Nursing Clinics of North America, vol. 40, n 3, p. 431-440.

DENHAM, A.R. (2008). "Rethinking historical trauma: Narratives of resilience», Transcultural Psychiatry, vol. 45, $\mathrm{n}^{\circ} 3$, p. 391-414.

DUMONT, C. (2007). «Ecological approaches to health: Interactions between humans and their environment", dans C. DUMONT et G. KIELHOFNER (dir.), Positive Approaches to Health, New York, Nova Science Publishers, p. 65-140.
DUMONT, C., P. FOUGEYROLLAS, M. GERVAIS et R. BERTRAND (2007). "Le processus d'adaptation des adultes présentant des séquelles de traumatisme craniocérébral », Revue canadienne d'ergothérapie, vol. 74, no 1, p. 48-60.

DUMONT, C. et F. RAINVILLE (2006). "Self, identity, and occupation», dans A.M. COLUMBUS (dir.), Advances in Psychology Research, vol. 45, New York, Nova Science Publishers, p. 181-227.

FERRER, L. et L.A.J. GOMEZ (2007). "Counseling bisexual Latinos: A minority within a minority », dans B.A. FIRESTEIN, Becoming Visible: Counseling Bisexuals Across the Lifespan, New York, Columbia University Press, ch. 13, p. 246-267.

FOUGEYROLLAS, P. (1996). «Les déterminants environnementaux de la participation sociale des personnes ayant des incapacités : le défi sociopolitique de la révision de la CIDIH», Revue canadienne de réadaptation, vol. $10, \mathrm{n}^{\circ} 2$, p. 147-160.

FOUGEYROLLAS, P. (2004). "Identité, différences corporelles et fonctionnelles, et processus de production du handicap sur le plan de la participation sociale», dans M. MERCIER (dir.), L'identité handicapée, Namur, Belgique, Facultés universitaires Notre-Dame de la Paix, coll. «Psychologie», no 5, p. 225-228.

FOUGEYROLLAS, P. et L. BEAUREGARD (2001). «Disability: An interactive personenvironment social creation", dans G.L. ALBRECHT et K. D. SEELMAN (dir.), Handbook of Disability Studies, Michael Burry - Sage, p. 171-194.

FOUGEYROLLAS, P., H. BERGERON, R. CLOUTIER, J. CÔTÉ et G. ST-MICHEL (1998A). Classification québécoise: Processus de production du handicap, Québec, CQCIDIH.

FOUGEYROLLAS, P., L. NOREAU, H. BERGERON, R. CLOUTIER, S.-A. DION et G. ST-MICHEL (1998B). «Social consequences of long term impairments and disabilities: Conceptual approach and assessment of handicap », International Journal of Rehabilitation Research, vol. 21, p. 127-141.

FOUGEYROLLAS, P. et L. NOREAU (2007). "L'environnement physique et social: une composante conceptuelle essentielle à la compréhension du processus de production du handicap. L'exemple des personnes ayant une lésion médullaire », dans J. BORIOLI et R. LAUB (dir.), Handicap: de la différence à la singularité. Enjeux au quotidien, Genève, Éd. Médecine et Hygiène, p. 47-69.

FOUGEYROLLAS, P., L. NOREAU et K. BOSCHEN (2002). "The interaction of environment with individual characteristics and social participation: Theoretical perspectives and application in persons with spinal cord injury ", Topics in Spinal Cord Injury Rehabilitation, vol. 7, $\mathrm{n}^{\circ}$ 3, p. 1-16.

GIBBONS, J.L. et B.K. ASHDOWN (2006). Emerging Adults in America: Coming on Age in the $21^{\text {st }}$ Century, Washington, DC, American Psychological Association, 341 p. 
GIBSON, E.C. (2002). "The impact of political violence: Adaptation and identity development in Bosnian adolescent refugees ", Smith College Studies in Social Work, vol. 73 , no 1 , p. 29-50.

GRAHAM-BERMANN, S.A. et H.M. HALABU (2004). "Fostering resilient coping in children exposed to violence: Cultural considerations », dans P.G. JAFFE, L.L. BAKER et A.J. CUNNINGHAM (dir.), Protecting Children from Domestic Violence: Strategies for Community Intervention, New York, Guilford Press, p. 71-88.

GRANT, G., P. RAMCHARAN et M. FLYNN (2007). "Resilience in families with children and adult members with intellectual disabilities: Tracing elements of a psycho-social model», Journal of Applied Research in Intellectual Disabilities, vol. 20, $\mathrm{n}^{\circ} 6$, p. 563-575.

HALL, J.C. et S.L. BOWIE (2007). "Introduction to research imperatives regarding African American human behaviour in the social environment", Journal of Human Behavior in the Social Environment, vol. 15, $\mathrm{n}^{\text {os }} 2-3$, p. 1-12.

HARRISON, T.W. (2003). "Adolescent homosexuality and concerns regarding disclosure ", Journal of School Health, vol. 73, $\mathrm{n}^{\circ} 3$, p. 107-112.

HOLLERAN, L.K. et M.A. WALLER (2003). "Sources of resilience among Chicano/a youth: Forging identities in the borderlands ", Child and Adolescent Social Work Journal, vol. 20, no 5, p. 335-350.

LEE, R.M. (2005). «Resilience against discrimination: Ethnic identity and othergroup orientation as protective factors for Korean Americans ", Journal of Counseling Psychology, vol. 52, $\mathrm{n}^{\circ}$ 1, p. 36-44.
NIKORA, L.W., M. RUA et N.T. AWEKOTUKU (2007). "Renewal and resistance: Moko in contemporary New Zealand», Journal of Community and Applied Social Psychology, vol. 17, $\mathrm{n}^{\circ}$ 6, p. 477-489.

NGUYEN-GILHAM, V., R. GIACAMAN, G. NASER et W. BOYCE (2008). "Normalising the abnormal: Palestinian youth and the contradictions of resilience in protracted conflict», Health and Social Care in the Community, vol. 16, nº 3, p. 291-298.

ONG, A.D., J.S. PHINNEY et J. DENNIS (2006). "Competence under challenge: Exploring the protective influence of parental support and ethnic identity in Latino college students", Journal of Adolescence, vol. $29, \mathrm{n}^{\circ} 6$, p. 961-979.

QUERIMIT, D.S. et L.C. CONNER (2003). "Empowerment psychotherapy with adolescent females of color ", Journal of Clinical Psychology, vol. 59, $\mathrm{n}^{\circ}$ 11, p. 1215-1224.

RIGGLE, E.D.B., J.S. WHITMAN, A. OLSON, S.S. ROSTOSKY et S. STRONG (2008). "The positive aspects of being a lesbian or gay man», Professional Psychology-Research and Practice, vol. 39, $\mathrm{n}^{\circ} 2$, p. 210-217.

ROSE, H.A., K.B. RODGERS et S.A. SMALL (2006). "Sexual identity confusion and problem behaviors in adolescents: A risk and resilience approach », Marriage and Family Review, vol. 40, n 2-3, p. 131-160.

ROUSSEAU, C., A. DRAPEAU et S. RAHIMI (2003). «The complexity of trauma response: A 4-year follow-up of adolescent Cambodian refugees", Child Abuse and Neglect, vol. 27, n 11 , p. 1277-1290.

SCHLOSSBERG, N.K. (2009). «Revitalizing retirement: Reshaping your identity, relationships, and purpose», dans Create a Lifetime of Possibilities, Washington, DC, American Psychological Association, p. 199-210.

SCOURFIELD, J., K. ROEN et L. MCDERMOTT (2008). «Lesbian, gay, bisexual and transgender young people's experiences of distress: Resilience, ambivalence and self-destructive behaviour", Health and Social Care in the Community, vol. 16, $\mathrm{n}^{\circ} 3$, p. 329-336.

SHIN, R., B. DALY et E. VERA (2007). "The relationships of peer norms, ethnic identity, and peer support to school engagement in urban youth », Professional School Counseling, vol. 10, no 4, p. 379-388.

SUNDAR, P. (2008). «To "brown it up" or to "bring down the brown": Identify and strategy in second-generation, south AsianCanadian youth", Journal of Ethnic and Cultural Diversity in Social Work, vol. 17, $\mathrm{n}^{\circ}$ 3, p. 251-278.

TUMMALA-NARRA, P. (2007). "Conceptualizing trauma and resilience across diverse contexts: A multicultural perspective », Journal of Aggression, Maltreatment and Trauma, vol. 14, $\mathrm{n}^{\circ}$ 1-2, p. 33-53.

UNGAR, M. et al. (2007). «Unique pathways to resilience across cultures ", Adolescence, vol. $42, \mathrm{n}^{\circ} 166$, p. 287-310.

WALLER, M.A. (2001). "Resilience in ecosystemic context: Evolution of the concept», American Journal of Orthopsychiatry, vol. 71, n 3, p. 290-297.

YICK, A.G. (2008). "A metasynthesis of qualitative findings on the role of spirituality and religiosity among culturally diverse domestic violence survivors", Qualitative Health Research, vol. 18, n 9, p. 1289-1306. 